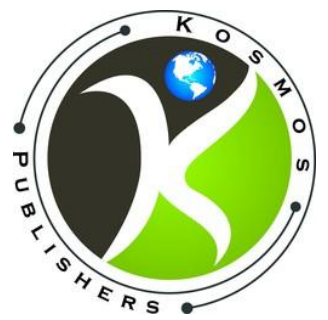

www.kosmospublishers.com contact@ kosmospublishers.com DOI: 10.37722/APHCTM.2021303

\title{
COVID-19 Infection in Nepal: Epidemiological Analysis from April 2020 to
} March 2021

\author{
Hai Le ${ }^{1,}$, Thomas Shen ${ }^{3}$, Sujan Marahatta ${ }^{4}$, Faith Boucher ${ }^{2}$ \\ ${ }^{I}$ Department of Orthopedic Surgery, Adult and Pediatric Spinal Surgery, UC Davis Medical Center, USA \\ ${ }^{2}$ University of California, Davis, Department of Public Health Sciences, Davis, California, USA \\ ${ }^{2}$ University of California, Davis, School of Medicine, Davis, California, USA \\ ${ }^{4}$ Manmohan Memorial Institute of Health Sciences, Kathmandu, Nepal
}

Received Date: September 06, 2021; Accepted Date: September 13, 2021; Published Date: September 21, 2021;

*Corresponding author: Hai Le, Department of Orthopedic Surgery, Adult and Pediatric Spinal Surgery, UC Davis Medical Center, USA. Email: haile@ucdavis.edu

Study Design: Retrospective epidemiological study

\section{Background}

In December 2019, SARS-CoV-2 emerged from Wuhan, China. A global pandemic called coronavirus disease 2019 (COVID-19) quickly unfolded, infecting >167 million people and causing >3.4 million deaths globally as of May 24, 2021. Before April 1, 2020, there were only five confirmed COVID19 cases in Nepal. Like many countries around the world, the COVID-19 situation quickly escalated in Nepal. The purpose of this study was to determine the trends in COVID-19 cases and deaths in Nepal from April 2020 to March 2021.

\section{Methods}

We utilized epidemiological data from daily Situation Reports published by the Ministry of Health and Population (MOHP) of Nepal. Data were extracted or calculated from April 1, 2020, to March 31, 2021. Primary variables of interest were national and provincial daily cases, total cases, daily deaths, and total deaths.

\section{Results}

Between April 1, 2020, to March 31, 2021, there were 277,304 cases. October 2020 had the highest monthly cases with 92,926 cases. During the one-year study period, the infection rate was 915 cases per 100,000 people. The largest number of single-day new cases occurred on October 21, 2020 , with 5,743 cases, which is calculated to 19 cases per 100,000 people. There were a total of 3,030 deaths. The largest daily new deaths occurred on November 4, 2020, with 43 cases. June 10, 2020, had the highest number of people in quarantine with 172,266 people. October 23,2020 , had the highest number of active cases with 46,329 cases. By March 31,2021 , the percent of mortality was $1.1 \%$, active infection was $0.5 \%$, and recovery was $98.4 \%$. Men comprised $64.9 \%$ of cases $(\mathrm{n}=179,886)$, while women comprised the remaining $35.1 \%(\mathrm{n}=97,418)$. The age groups $21-30(26.8 \%), 31-40$ (26.2\%), and 41-50 (16.7\%) were most affected by COVID19. Of the 277,304 cases, $55.1 \%$ occurred in Bagmati. Bagmati had the largest infection rate of 2,764 cases per 100,000 people, while Province 2 had the lowest infection rate of 387 cases per 100,000 people.

\section{Conclusions}

Nepal had lower COVID-19 infection and case-fatality rates compared to other countries most affected by the pandemic. This was due to several factors, most notably early implementation of strict lockdown measures and closing of international borders on March 24, 2020, after the second confirmed COVID-19 case. As lockdown restrictions were lifted on July 7, 2020, COVID-19 cases and deaths in Nepal rose rapidly. As vaccination began on January 27, 2021, cases started to slow down until the most recent outbreak coinciding 
with the second wave in its neighboring country, India. Now, infection and case-fatality rates in Nepal are at an all-time high, prompting further lockdowns on April 29, 2021.

\section{Introduction}

In December 2019, a novel coronavirus responsible for coronavirus disease 2019 (COVID-19) called SARS-CoV-2 emerged from Wuhan, China. ${ }^{1}$ The World Health Organization (WHO) declared the COVID-19 outbreak a Public Health Emergency of International Concern on January 30, 20202, and upgraded it as a global pandemic on March 11, 2020., Given the coronavirus' high infectivity through airborne transmission, a contemporary global pandemic quickly unfolded and has since placed significant burden on healthcare systems worldwide. As of May 24, 2021, there have been $>167$ million COVID-19 cases and >3.4 million COVID-19 related deaths globally. ${ }^{5}$

The first confirmed case in Nepal was a 31-year-old Nepalese student who was pursuing his $\mathrm{PhD}$ abroad in Wuhan. ${ }^{2}$ He developed symptoms on January 3, 2020, and returned to Nepal on January 9, 2020, for winter holidays. He presented to the Sukraraj Tropical and Infectious Disease Hospital (STIDH) in Kathmandu on January 13, 2020. Given his recent travel history, he was isolated at STIDH for four days and was subsequently discharged home for selfquarantine. ${ }^{2}$ At the time, Nepal did not have its own testing center; therefore, the student's throat swab sample was sent overseas to the WHO Collaborating Center in Hong Kong. The test returned positive on January 23, 2020, ten days after sample collection. The next seven COVID-19 cases were Nepalis who had returned to Nepal after traveling abroad, including one returning from France, two from United Arab Emirates (UAE), two from Belgium, and two from India. ${ }^{6}$ The first confirmed local transmission COVID-19 case occurred on April 4, 2020, in the Sudurpaschim province.

Nepal's COVID-19 response has been under the leadership of the Ministry of Health and Population (MOHP). Since its first confirmed cases, like other countries worldwide, Nepal has had a rapid surge in total cases and deaths from COVID$19 .{ }^{6}$ In response, Nepal mandated a countrywide lockdown on March 24, 2020. ${ }^{4}$ The National Public Health Laboratory (NPHL) in Kathmandu was the first center to perform diagnostic testing for Nepalese citizens. ${ }^{4}$ As of April 13, 2021, the country has expanded to more than 67 laboratories with capability to perform real-time polymerase chain reaction (RT-PCR) diagnostic testing.

As the COVID-19 pandemic pushes past the one-year mark with no slowing in sight, we utilized epidemiological data provided by MOHP to evaluate how Nepal has responded to COVID-19. The purpose of this study was to determine the trends in COVID-19 cases and deaths in Nepal from April 2020 to March 2021. The impact of COVID-19 was also compared among the seven provinces of Nepal.

\section{Methods}

\section{Data Collection}

Since January 28, 2020, MOHP has published a daily Situation Report which provides an update on the COVID-19 pandemic in Nepal. The Situation Reports contain national and provincial epidemiological data. For this study, the Situation Reports from April 1, 2020, to March 31, 2021, were downloaded from the Government of Nepal's Google Drive repository for analysis. April 1, 2020, was chosen as the initial date because data collected from earlier Situation Reports were limited and inconsistent. Moreover, prior to April 1, 2020, there were only five confirmed COVID-19 cases in Nepal. The Situation Reports were cross-referenced with the MOHP COVID-19 online dashboard ${ }^{7}$ as well as Worldometer $^{8}$ to ensure accuracy of data. The data were summarized for the entire country of Nepal. In addition, the impact of COVID-19 was compared between the seven provinces of Nepal, which include Province 1, Province 2, Bagmati, Gandaki, Lumbini, Karnali, and Sudurpashchim. According to MOHP, sex was categorized dichotomously as male or female. Age was grouped into the following age brackets: 0-10, 11-20, 21-30, $31-40,41-50,51-60,61-70,71-80$, and 80+ years old.

\section{Variables of Interest}

Variables of interest were directly collected or calculated from the Situation Reports. Daily cases were COVID-19 cases that were diagnosed over the past 24 hours. Total cases were cumulative COVID-19 cases. Active cases included patients who had not yet recovered from COVID-19. Recovered cases were patients who had completely recovered from COVID-19 without symptoms. Daily deaths were new mortality cases over the past 24 hours. Total deaths were cumulative COVID19 related mortality cases. People in quarantine were the number of citizens who were separated if they had close contact with someone with COVID-19. Total RT-PCR tests performed were also collected.

The monthly cases were calculated for sex and age group. Rates were calculated using national and provincial population data. As of April 21, 2021, the population of Nepal was $30,313,948$ people. ${ }^{9}$ Provincial population was derived from the 2011 Census. ${ }^{10}$

\section{Results}

\section{National Data}

Between April 1, 2020, to March 31, 2021, there were 277,304 COVID-19 cases in Nepal. Prior to April 1, 2020, there were only 5 cases. Thus, the total number of COVID-19 cases was 277,309 as of March 31, 2021 (Figure 1). October 2020 had the highest monthly COVID-19 cases with 92,926 cases. Over the one-year study period, the COVID-19 infection rate was 915 cases per 100,000 people in Nepal. 


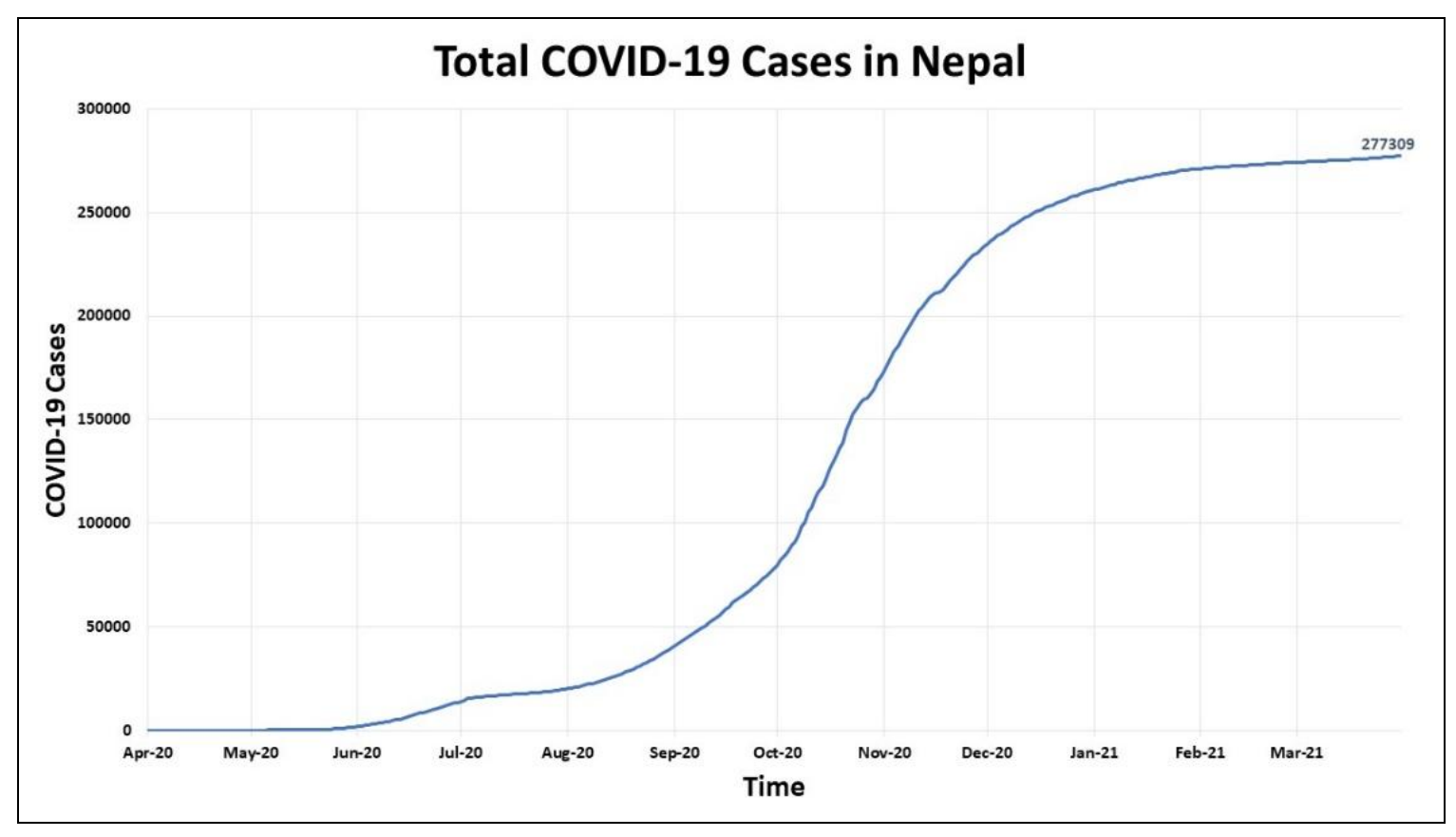

Figure 1. Total COVID-19 Cases in Nepal from April 1, 2020, to March 31, 2021.

(Figure 2) summarizes daily new cases in Nepal. The largest single-day new cases occurred on October 21, 2020, with 5,743 cases, which is calculated to 19 cases per 100,000 people.

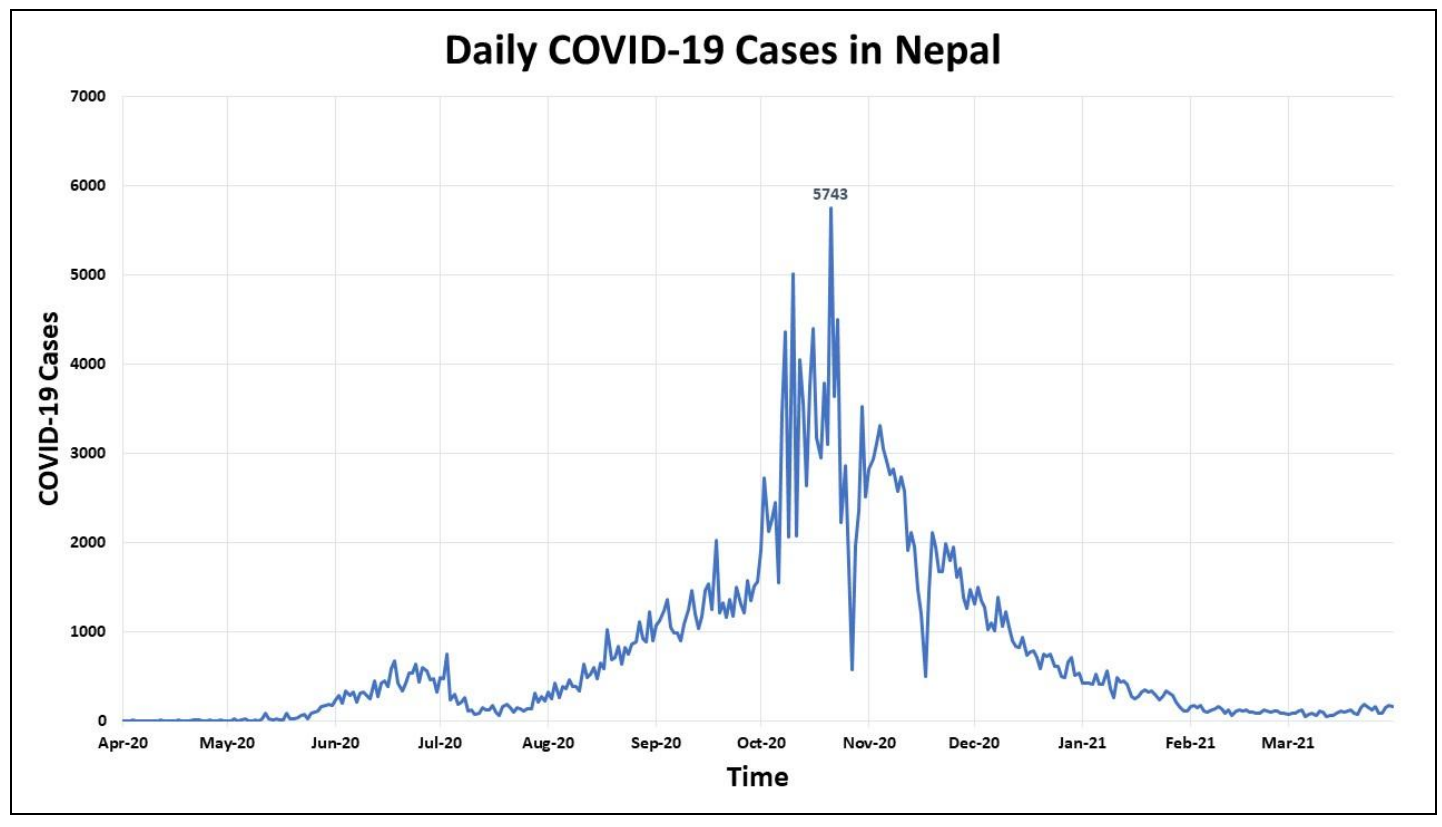

Figure 2. Daily COVID-19 Cases in Nepal from April 1, 2020, to March 31, 2021.

Nepal had its first COVID-19 related mortality on May 16, 2020. By March 31, 2021, there were a total of 3,030 deaths (Figure 3). 


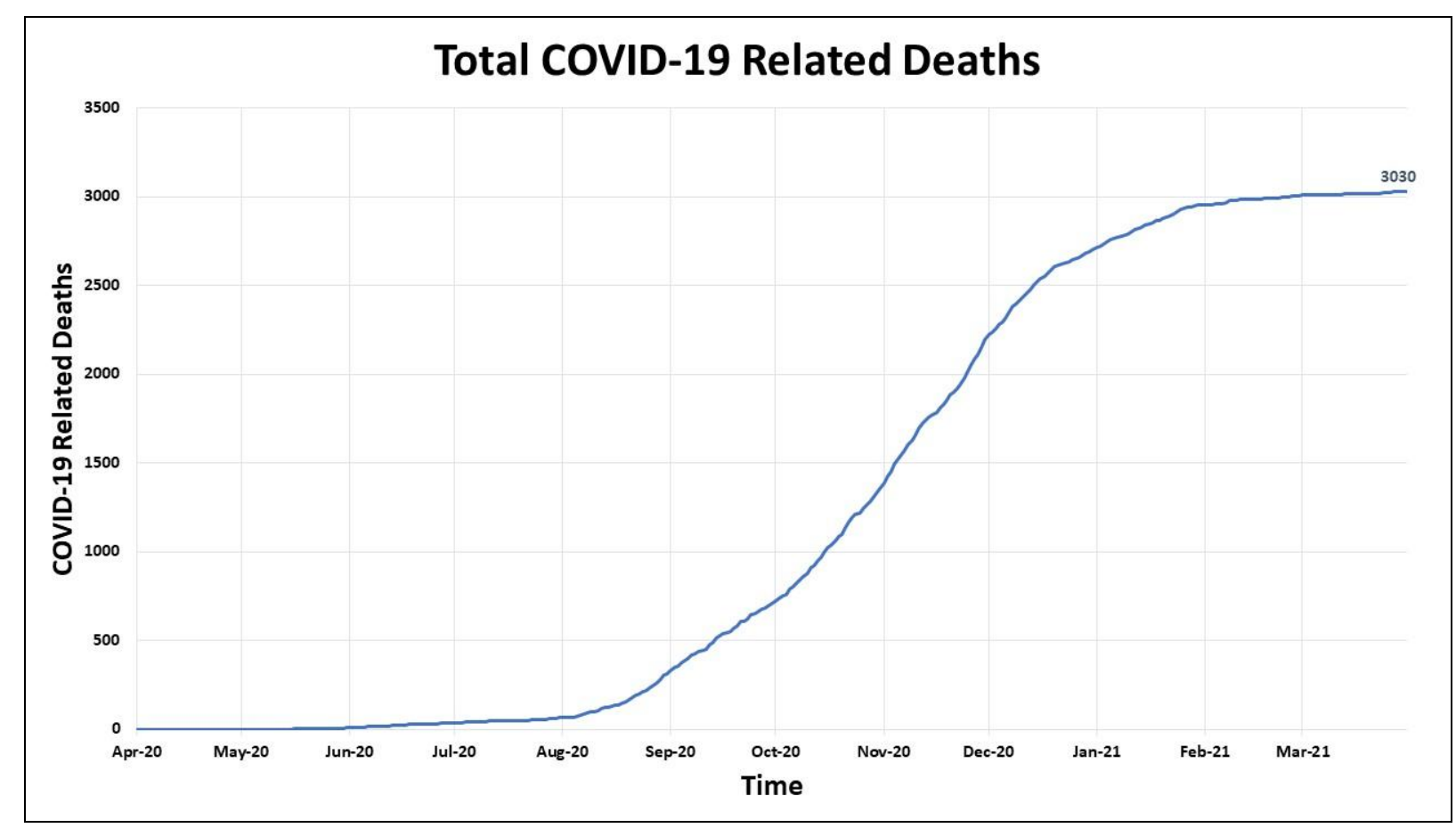

Figure 3. Total COVID-19 Related Deaths in Nepal from April 1, 2020, to March 31, 2021.

The largest daily new deaths occurred on November 4, 2020, with 43 cases (Figure 4).

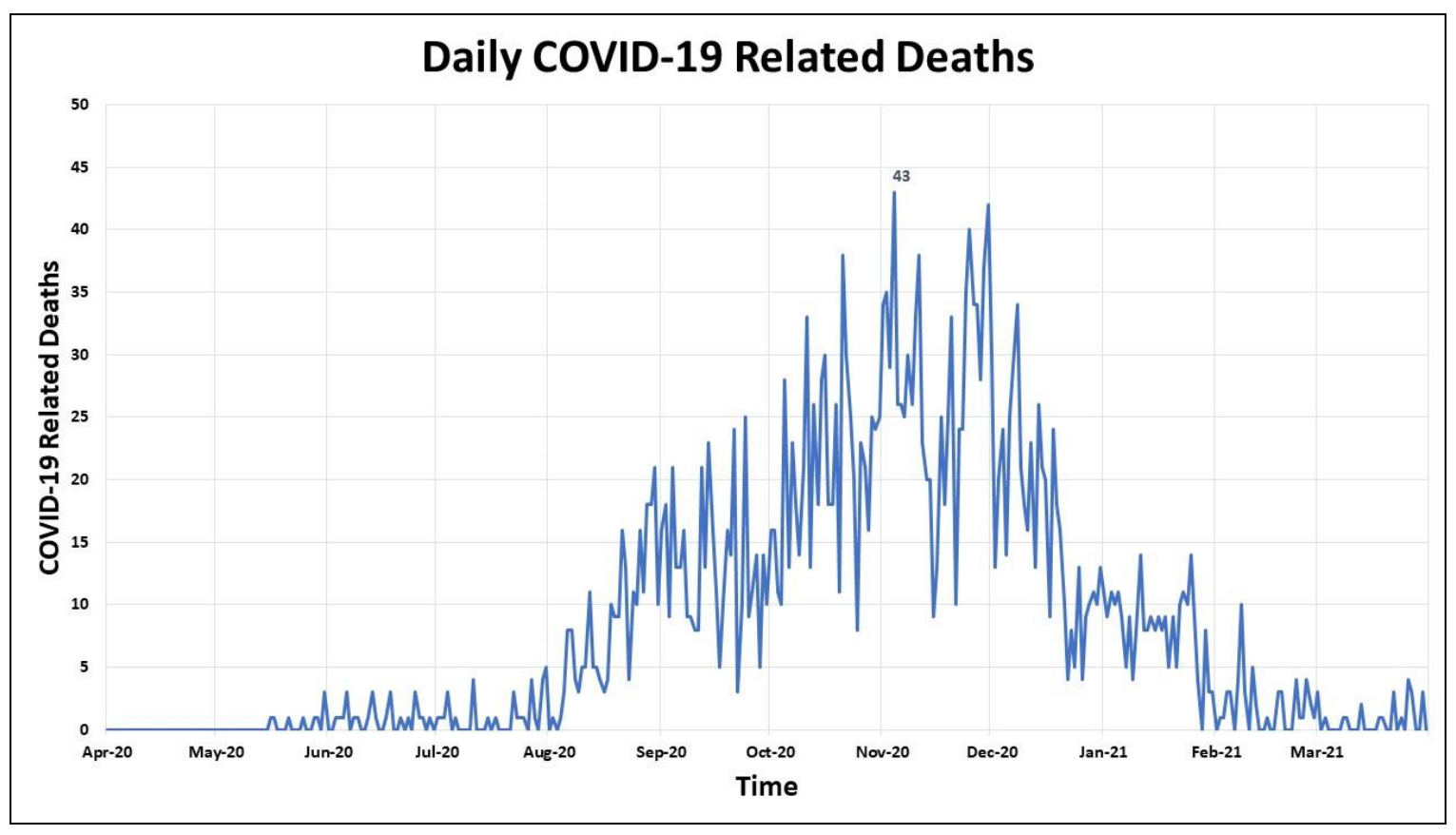

Figure 4. Daily COVID-19 Related Deaths in Nepal from April 1, 2020, to March 31, 2021.

(Figure 5) demonstrates total RT-PCR tests performed. As of March 31, 2021, there were 2,271,327 RT-PCR tests that had been performed, or 77,953 RT-PCR tests per million people. 


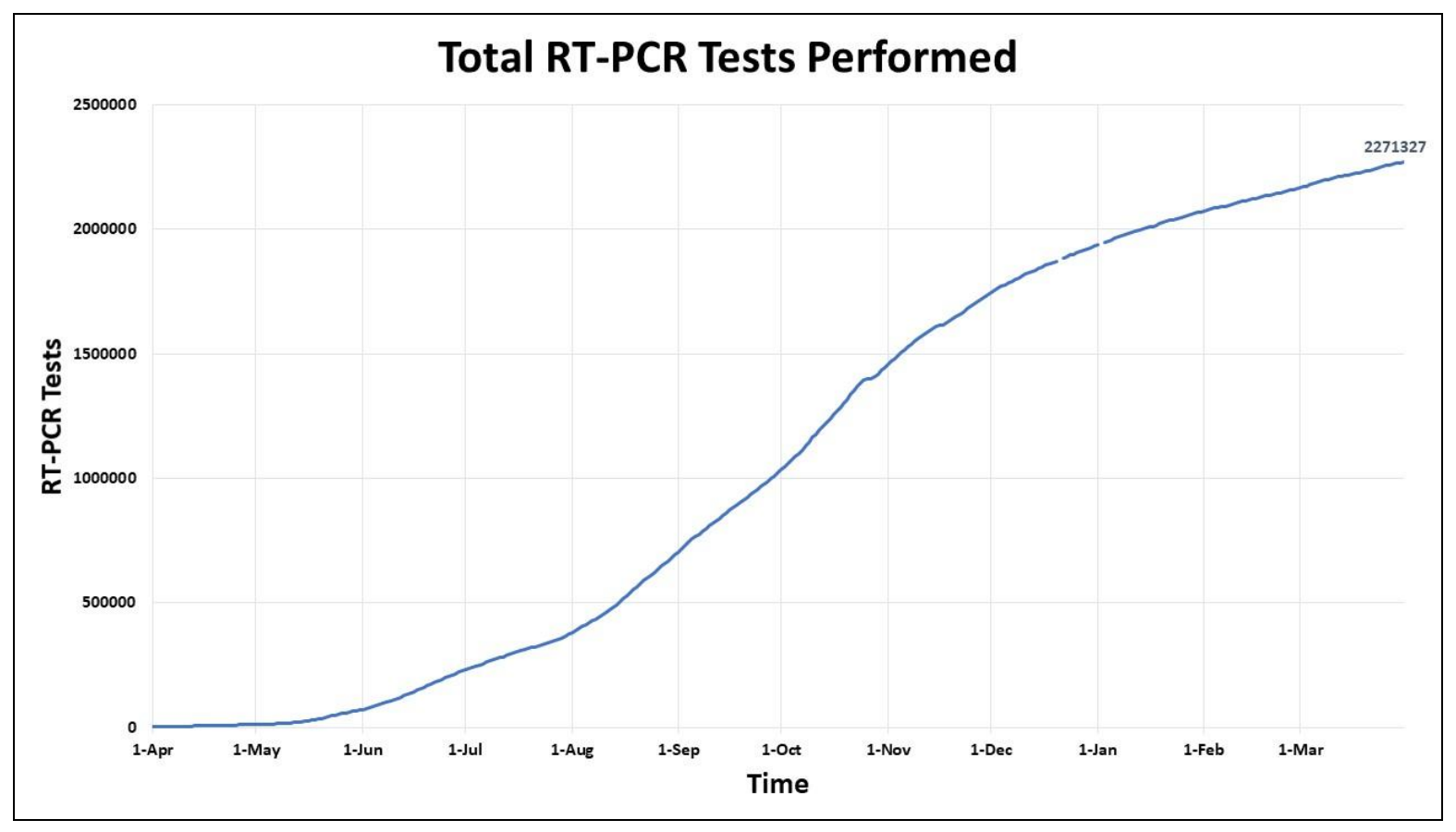

Figure 5. Total RT-PCR Tests Performed in Nepal.

(Figure 6) demonstrates active cases and people in quarantine. June 10, 2020, had the highest number of people in quarantine with 172,266 people. October 23, 2020, had the highest number of active cases with 46,329 cases.

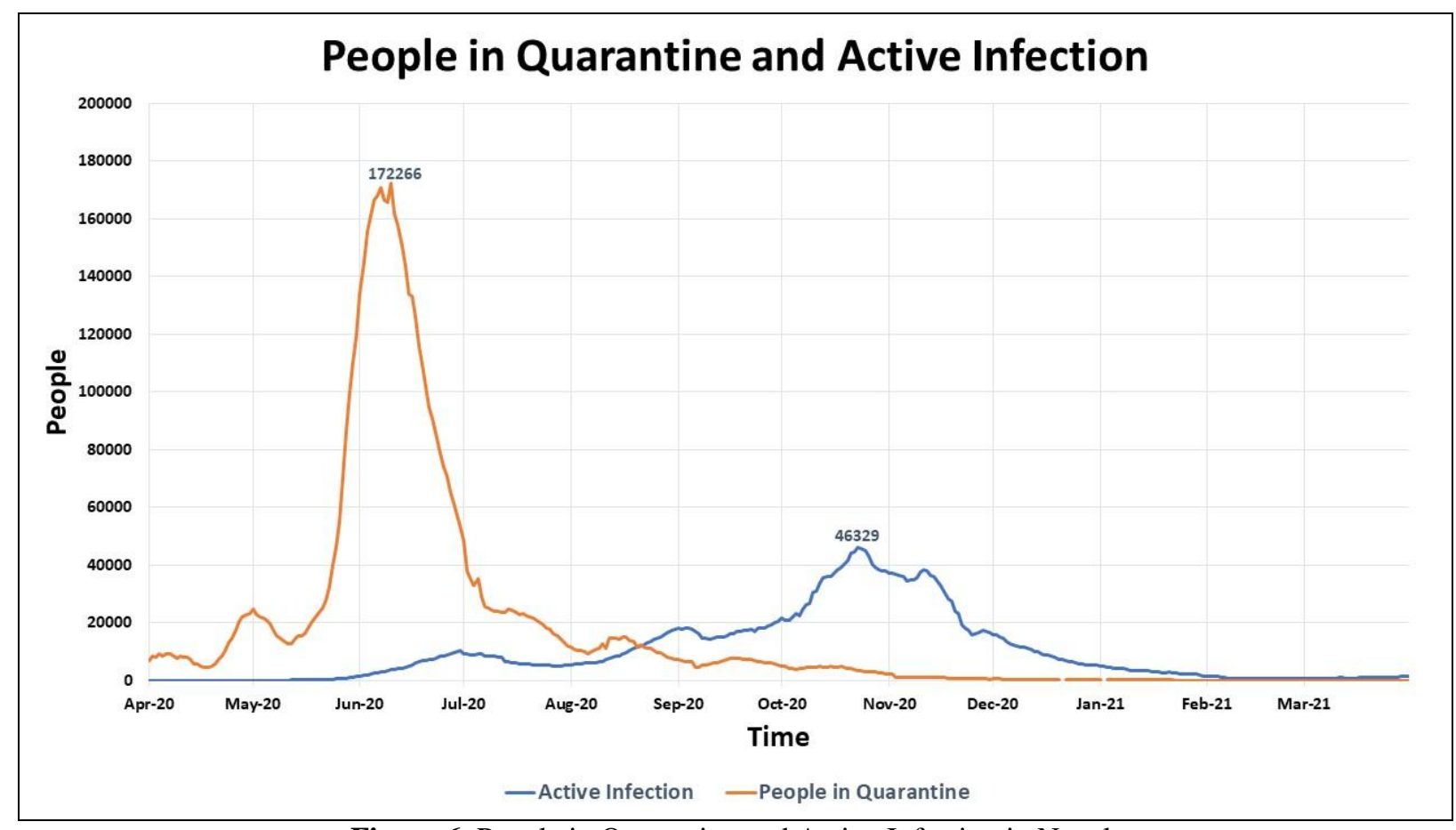

Figure 6. People in Quarantine and Active Infection in Nepal.

(Figure 7) displays percentage of COVID-19 recovery. 


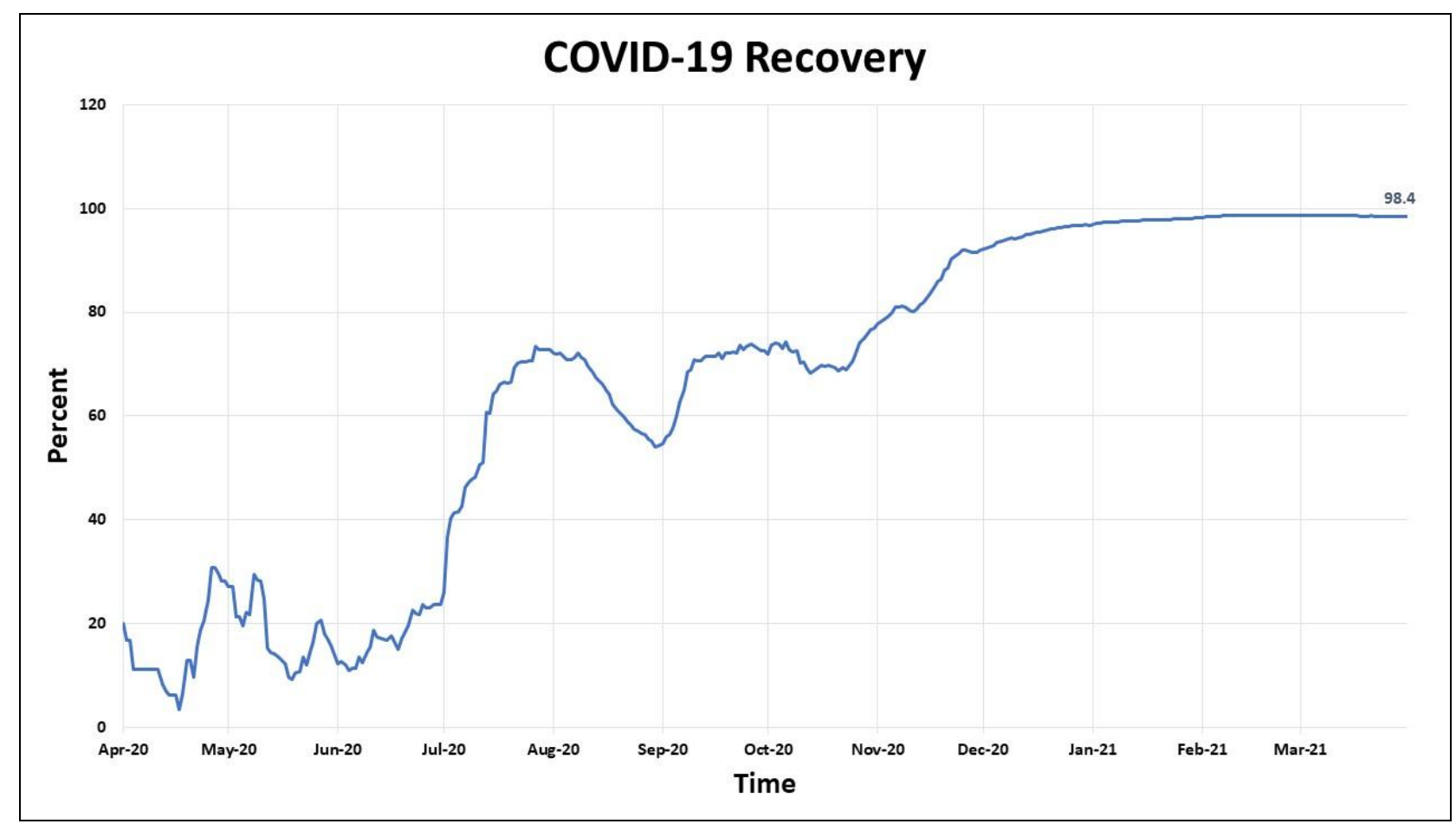

Figure 7. Percent of COVID-19 Recovery in Nepal.

By March 31, 2021, the percentage of mortality (case mortality rate) was $1.1 \%$, active infection was $0.5 \%$, and recovery was $98.4 \%$ (Figure 8).

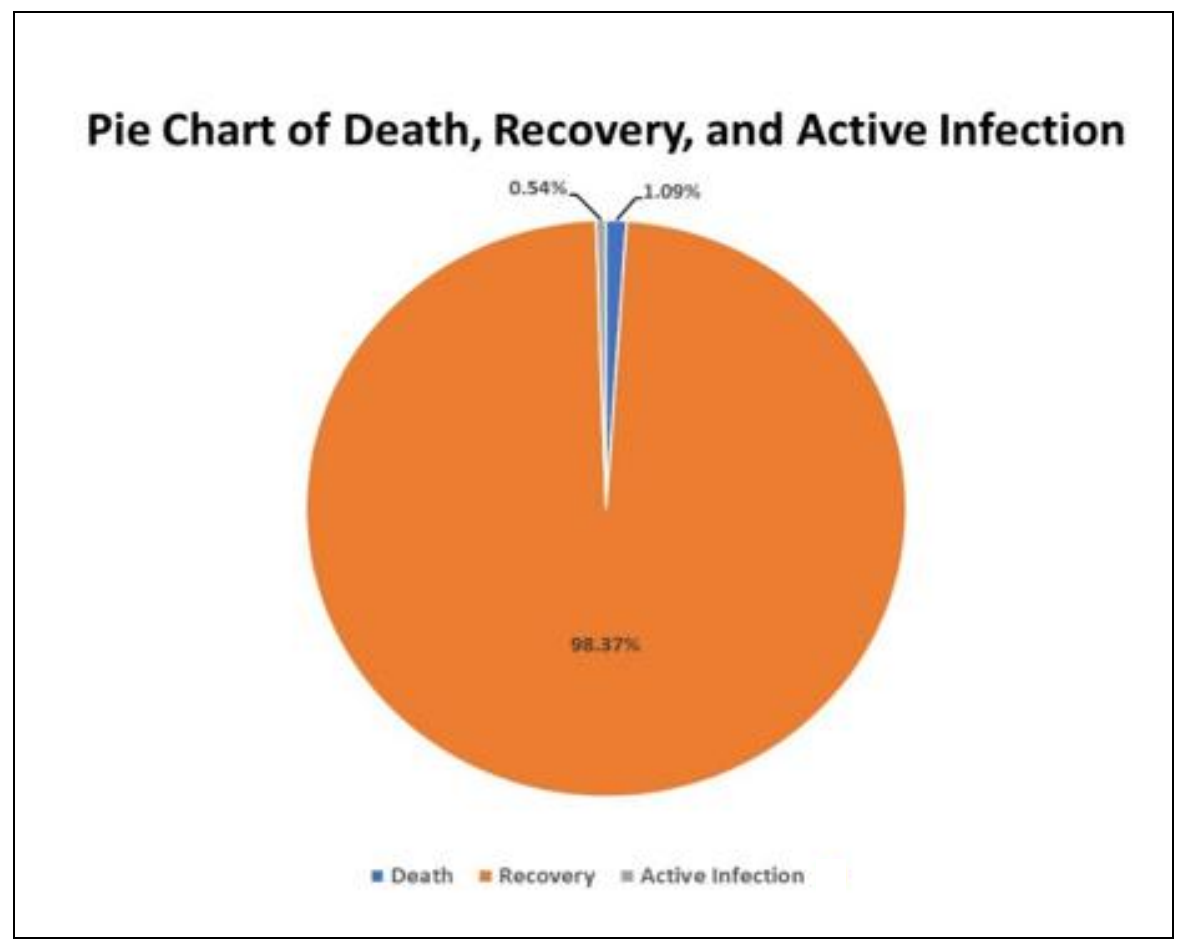

Figure 8. Pie Chart of COVID-19 Death, Recovery, and Active Infection.

Men comprised $64.9 \%$ of cases $(n=179,886)$, while women comprised the remaining $35.1 \%(n=97,418)$. The number of cases by age group and sex is summarized in (Figure 9). The age groups 21-30 (26.8\%), 31-40 (26.2\%), and 41-50 (16.7\%) were most affected by COVID-19. Higher proportions of men had COVID-19 compared to women in all age groups. 


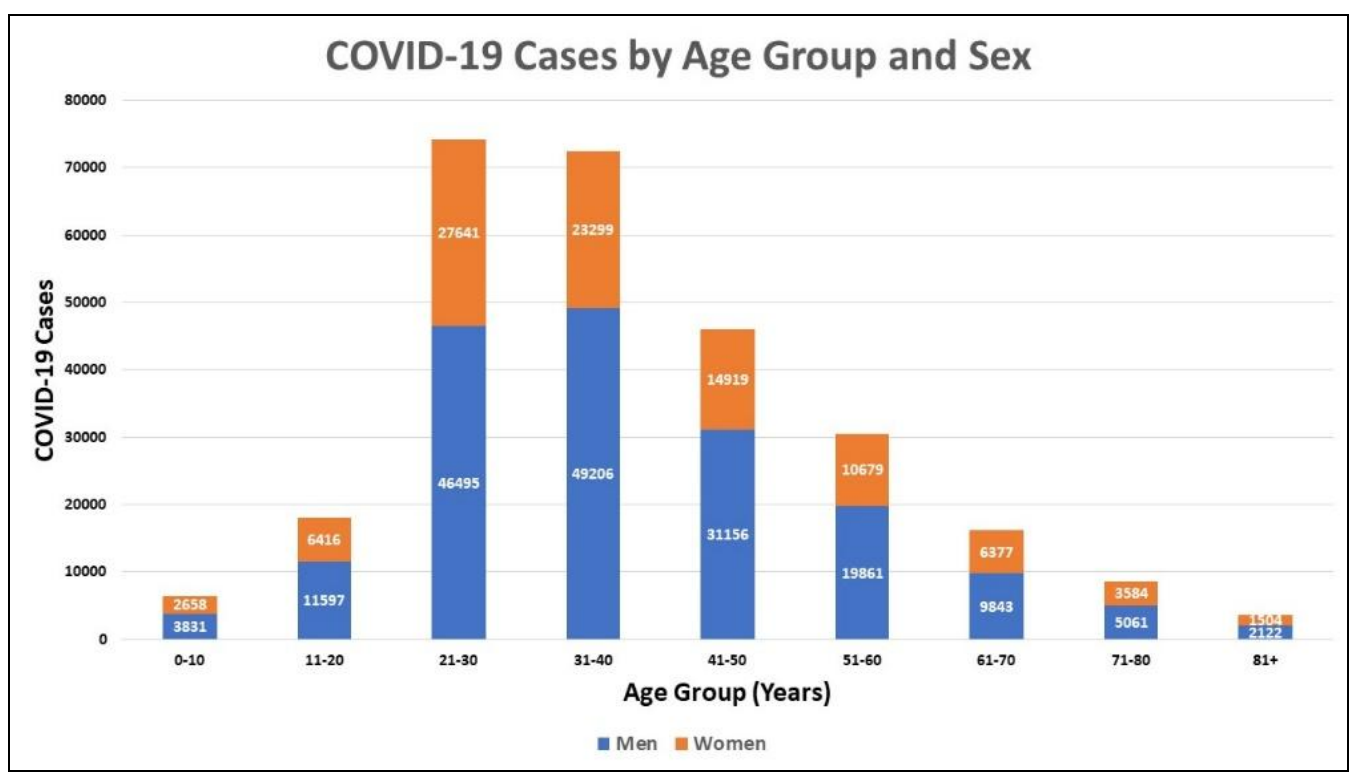

Figure 9. COVID-19 Cases by Age Group and Sex.

\section{Provincial Data}

Of the 277,304 cases during the study period, 55.1\% of cases occurred in Bagmati (Figure 10).

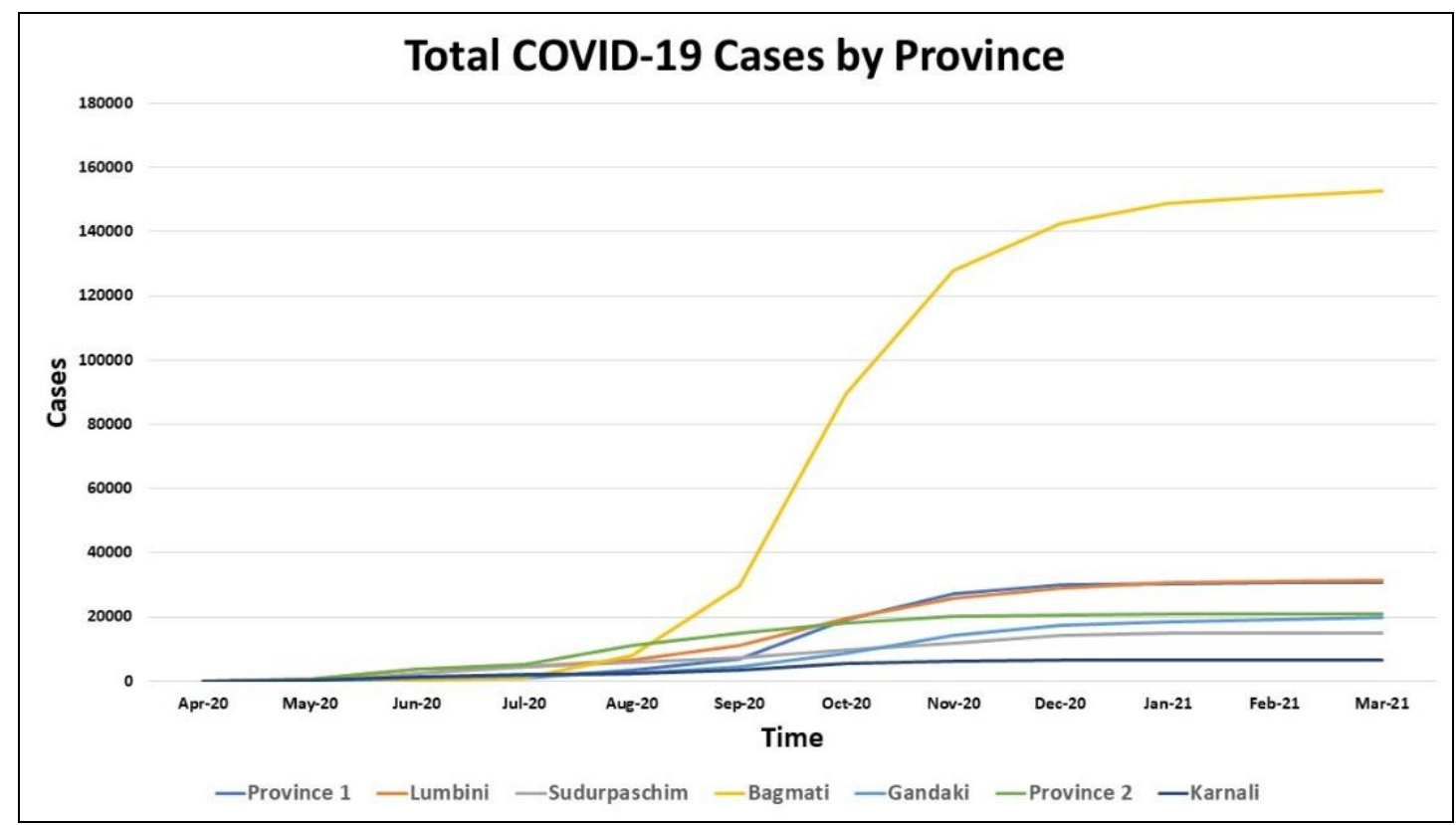

Figure 10. Total COVID-19 Cases by Province.

Bagmati had the largest infection rate of 2,764 cases per 100,000 people, while Province 2 had the lowest infection rate of 387 cases per 100,000 people (Figure 11). 


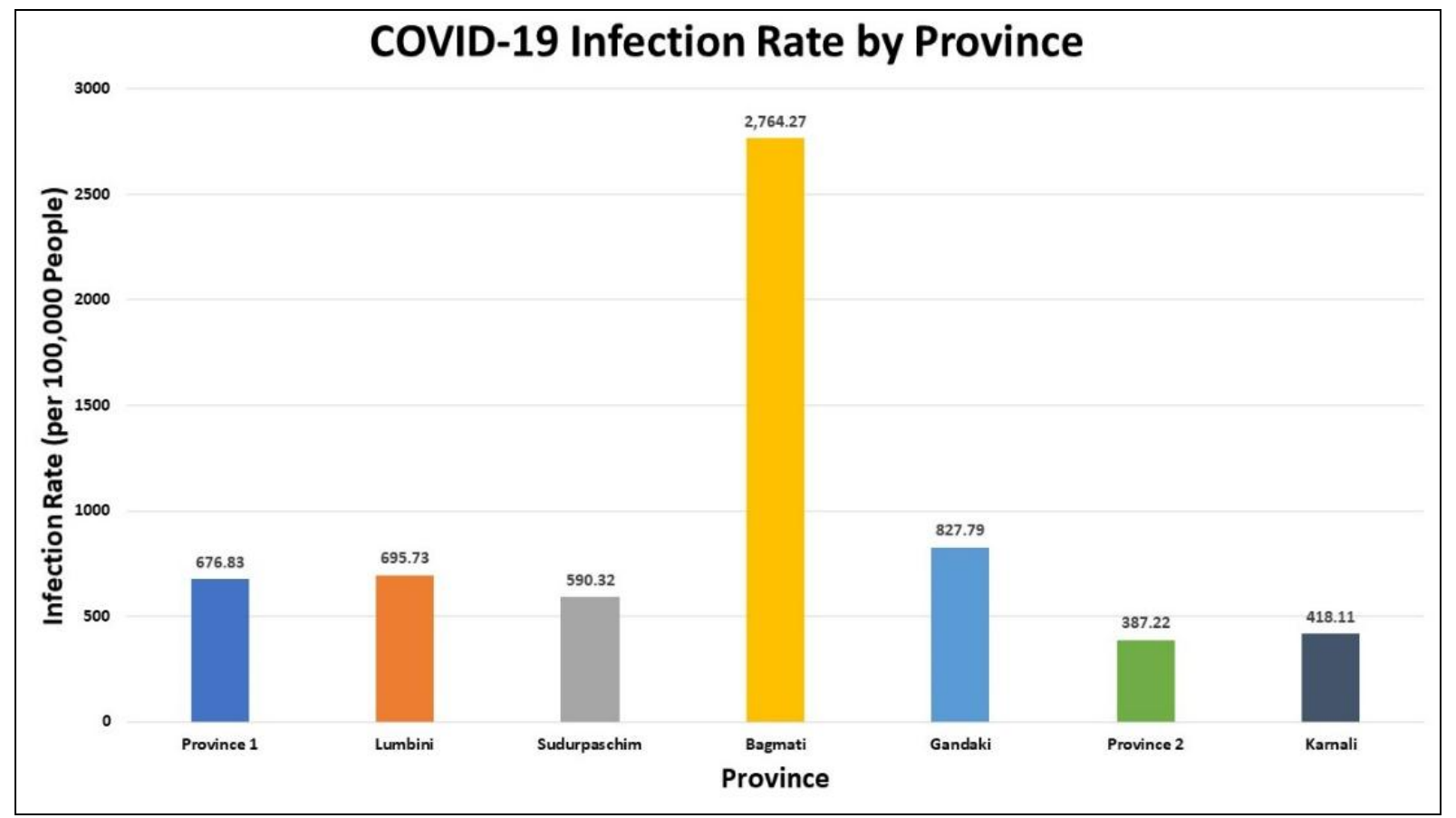

Figure 11. COVID-19 Infection Rate by Province.

Monthly COVID-19 cases by province are summarized in (Figure 12).

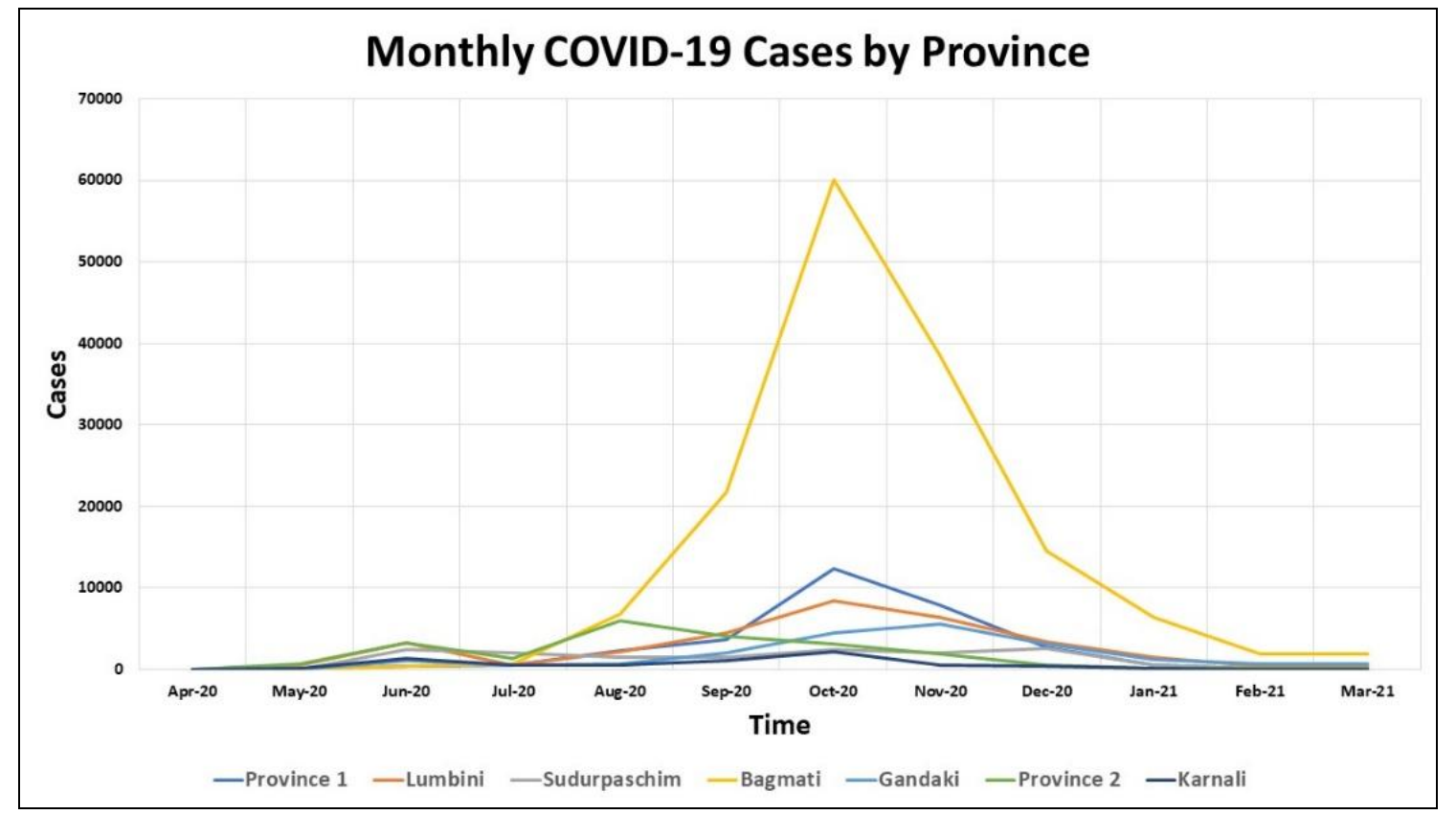

Figure 12. Monthly COVID-19 Cases by Province.

\section{Discussion}

This is the largest epidemiological study of COVID-19 cases and mortality in Nepal, and one of the first studies to examine variability in COVID-19 cases and mortality between the country's seven provinces. In our study, we found that Nepal had lower COVID-19 infection and case-fatality rates compared to other countries most affected by the pandemic. In particular, the infection rate was 915 cases per 100,000 people, while the case-fatality rate was $1.1 \%$. For comparison, the United States has an infection rate of 10,054 per 100,000 people and a case-fatality rate of $1.8 \%$. Nepal's bordering countries China and India have case-fatality rates of $4.7 \%$ and $1.1 \%$, respectively. ${ }^{11}$ Lower rates of infection and casemortality observed in Nepal were due to several important 
factors. Most importantly, Nepal implemented strict lockdown measures and closing of international borders on March 24, 2020, after the second confirmed COVID-19 case. Furthermore, quarantine centers were quickly developed and enforced by the Office of the Prime Minister and Council of Ministers on March 29, 2020, to mitigate infection risks. Criteria for quarantine included a) anyone who had come in contact with an infected individual, b) anyone who had returned from a country severely affected by COVID-19, or c) anyone who had been in transit in a country severely affected by COVID$19 .{ }^{12}$

As lockdown restrictions were lifted on July 7, 2020, COVID-19 cases and deaths in Nepal rose rapidly. Like many countries across the world, nationwide lockdown severely affected Nepal's economy, prompting the need to lift restrictions. Nepal utilized the lockdown to develop the COVID-19 Crisis Management Centre, train healthcare workers, and expand laboratory capacity. ${ }^{12}$ Therefore, the rise in COVID-19 cases during the summer of 2020 was likely due to lifting of lockdown restrictions as well as the increase in testing capacity. This is supported by similar trajectories between total RT-PCR tests performed (Figure 5) and total COVID-19 cases (Figure 2). As vaccination began on January 27, 2021, cases started to slow down until the most recent outbreak coinciding with the second wave in its neighboring country, India. Now, infection and case-fatality rates in Nepal are at an all-time high, prompting lockdown of Kathmandu and nearby districts on April 29, 2021. During this second wave, the daily COVID-19 cases peaked at 9,317 cases, far exceeding the peak of 5,743 daily cases during its first wave. The daily mortality similarly peaked on May 11, 2021, with 225 cases, as compared to 43 daily deaths on November 4, $2020 .^{7}$ The similarities between outbreaks in India and Nepal are in part due to mobility between the two countries, largescale religious gatherings, and low vaccine coverage. ${ }^{13}$

There are several important findings from our study that are worth highlighting. In Nepal, men (64.9\%) were more affected than women $(35.1 \%)$. In contrast, globally, COVID19 infection is distributed fairly evenly between men $(50.9 \%)$ and women (49.1\%). ${ }^{14}$ In Nepal, COVID-19 infection was more common in younger patients between the age of 21-50 years. We hypothesize these findings to be due to the higher proportion of Nepalese men who are labor migrants. Labor migrants are particularly at high risk for COVID-19 infection. According to Nepal Labour Migration Report 2020, female migrant workers only comprised $8.5 \%$ of total migrant workers. Furthermore, the majority of Nepali migrant workers were between age 18-45 years. ${ }^{15}$ Interestingly, most Nepali migrant workers work in India because visa and work-permit are not required by law. ${ }^{15}$ An estimated 3 million Nepali migrant workers work in India. ${ }^{16}$ This could explain the similar trends in COVID-19 cases between the two countries.

Our study has unique strengths and limitations. The data were derived directly from Situation Reports by MOHP, and data points were checked with online COVID-19 dashboards to ensure accuracy in reporting. Thus, this study represents the largest epidemiological study of COVID-19 cases and deaths in Nepal over a one-year time period. Granular data on each case were limited and therefore we could not assess comorbidities and other risk factors for COVID-19 illness. Furthermore, mortality data and vaccination data were not readily available for analysis through the Situation Reports provided by MOHP.

At the conclusion of our study, Nepal is amidst its second wave of the COVID-19 pandemic. While Kathmandu and surrounding districts have received lockdown restrictions, the government needs to enforce a nationwide lockdown to slow the rapid surge in COVID-19 cases and deaths. International borders, especially between Nepal and India, need to temporarily close until the country broadens COVID-19 vaccine distribution. Sadly, the United Kingdom (UK) variant B.1.1.7 of SARS-CoV-2 has already been confirmed in Nepal. ${ }^{17}$ Recently, genome sequencing of samples collected from 35 patients revealed that the B.1.617.2 variant was detected in $97 \%$ of samples, while the B.1.617.1 variant was detected in the remaining $3 \%$ of samples. ${ }^{7}$ Since Nepal is resource-limited, the country has been receiving vaccines donated from India as a humanitarian aid. However, as India battles its own COVID-19 outbreak, the exports of AstraZeneca vaccines by India to Nepal have been halted, thus further hampering Nepal's vaccination drive. ${ }^{19}$ The Government of Nepal needs to improve contact tracing, expand laboratory capacity, strengthen its vaccination efforts, and immediately impose a nationwide lockdown in order to flatten the curve of this second COVID-19 wave.

\section{References}

1. Zhu N, Zhang D, Wang $W$ et al. (2020) A novel coronavirus from patients with pneumonia in China, 2019. N Engl J Med. 382:727-733.

2. Shrestha R, Shrestha S, Khanal P et al. (2020) Nepal's first case of COVID-19 and public health response. J Travel Med. 27:taaa024.

3. Cucinotta D, Vanelli M (2020) WHO declares COVID-19 a pandemic. Acta Biomed. 91:157-160.

4. Piryani RM, Piryani S, Shah JN (2020) Nepal's response to contain COVID-19 infection. J Nepal Health Res Counc. 18:128-134.

5. COVID-19 Dashboard by the Center for Systems Science and Engineering (CSSE) at Johns Hopkins University (JHU). Coronavirus Resource Center. Last updated May 24, 2021. Accessed May 2024, 2021.

6. Panthee B, Dhungana S, Panthee N et al. (2020) COVID19: the current situation in Nepal. New Microbes New Infect. 37:100-737.

7. Nepal Last 24 Hours Update. Government of Nepal. Ministry of Health and Population. Last updated May 24, 2021. Accessed May 24, 2021.

8. Nepal Coronavirus Cases. Worldometer. Last updated May 24, 2021. Accessed May 24, 2021.

9. National Census 2021. Government of Nepal. National Planning Commission. Central Bureau of Statistics. Last updated April 21, 2021. Accessed April 21, 2021. 


\section{Analysis from April 2020 to March 2021}

10. Population by Province. Government of Nepal. National Planning Commission. Central Bureau of Statistics. Last updated April 21, 2021. Accessed April 21, 2021.

11. Mortality in the most affected countries. Johns Hopkins University. Last updated May 19, 2021. Accessed May 19, 2021.

12. Responding to COVID-19. Health sector preparedness, response and lessons learnt. Government of Nepal. Ministry of Health and Population. Unknown last updated. Accessed May 19. 2021.

13. Hollingsworth J, Jeong $S$. Nepal's cases skyrocket, prompting concern the country's outbreak could mimic India's. CNN. Last updated May 6, 2021. Accessed May 19, 2021.
14. Gender and COVID-19. COVID-19 and gender monitor. UN Women. Last updated June 26, 2020. Accessed May 19, 2021.

15. Nepal Labour Migration Report 2020. Government of Nepal. Ministry of Labour, Employment and Social Security. Accessed May 19, 2021.

16. Kunwar LS (2015) Emigration of Nepalese people and its impact. Economic Journal of Development. 19:77-82.

17. Sah R, Khatiwada AP, Shrestha S et al. (2021) COVID-19 vaccination campaign in Nepal, emerging UK variant and futuristic vaccination strategies to combat the ongoing pandemic. Travel Med Infect Dis. 41:102037.

18. Kansakar S, Dumre SP, Raut A et al. (2021) From lockdown to vaccines: challenges and response in Nepal during the COVID-19 pandemic [published online ahead of print, 2021 Apr 28]. Lancet Respir Med.

Citation: Le H, Shen T, Marahatta S, Boucher F (2021) COVID-19 Infection in Nepal: Epidemiological Analysis from April 2020 to March 2021. Adv Pub Health Com Trop Med: APCTM-130. 